\title{
Engineering safety recommendations: Results from a survey in aviation
}

\author{
N. Karanikas \\ Aviation Academy, Faculty of Technology, Amsterdam University of Applied Sciences, The Netherlands
}

\begin{abstract}
Taking into account the lack of uniform guidelines for the design and classification of safety recommendations, a relevant framework was developed according to academic and professional literature. The framework includes nine design criteria for recommendations, it incorporates classifications of their scope and expected effectiveness, and it was used to perform a questionnaire survey across aviation professionals involved in the generation of safety recommendations. The goal of the survey was to capture (1) whether practitioners are knowledgeable about the design criteria, (2) the degree to which they apply those criteria along with corresponding reasons, (3) perceptions of the expected effectiveness of types of controls introduced through recommendations, (4) the frequency of generating each control type and respective explanations, and (5) the extent to which practitioners focus on each of the categories of recommendations' scope and the relevant reasons. Overall, the results showed: an adequate level of knowledge of the design criteria; a strong positive association of the knowledge on a particular criterion with the degree of its implementation; a variety of frequencies the recommendations are addressed to each of the scope areas; a reverse order of perception of the expected effectiveness of control types compared to the literature suggestions. A thematic analysis revealed a broad spectrum of reasons about the degree to which the design criteria are applied, and the extent to which the various types of recommendations are generated. The results of the survey can be exploited by the aviation sector to steer its relevant education and training efforts and assess the need for influencing the direction safety recommendations are addressed. Similar research is suggested to be conducted by organizations and regional and international agencies of any industry sector by ensuring a larger sample.
\end{abstract}

\section{INTRODUCTION}

Safety investigations play a crucial role in safety improvements especially because they lead to the formulation of recommendations to eliminate or mitigate identified problems with the scope to prevent similar occurrences in the future. To date, although in aviation there are established guidelines for the conduction of a safety investigation (ICAO, 2003, 2008, 2011, 2015), there is yet little guidance about the design of safety recommendations (Pooley, 2013).

To fill this gap, researchers and students from the Aviation Academy of the Amsterdam University of Applied Sciences (Zonneveld, 2016, De Vos, 2016, Kiefer, 2016) reviewed academic and professional literature and proposed a relevant framework that includes design criteria (Table 1), their scope (Table 2) and expected effectiveness (Table 3).

The particular framework was used to perform a questionnaire survey across safety practitioners to explore the extent to which the framework aspects are known and applied, and reveal any underlying reasons.
Table 1. Design criteria for safety recommendations.

\begin{tabular}{|c|c|c|}
\hline Criterion & Brief explanation & Literature* \\
\hline Specific & $\begin{array}{l}\text { Addresses a } \\
\text { particular problem }\end{array}$ & $\begin{array}{l}\text { (Haughey, 2014, } \\
\text { Gregson, } \\
\text { 2017) }\end{array}$ \\
\hline Measurable & $\begin{array}{l}\text { Allows monitoring of } \\
\text { its implementation }\end{array}$ & (Haughey, 2014) \\
\hline Assigned & $\begin{array}{l}\text { Addressed to specific } \\
\text { responsible agent(s) }\end{array}$ & $\begin{array}{l}\text { (Haughey, 2014, } \\
\text { Gregson, } \\
\text { 2017) }\end{array}$ \\
\hline Realistic & $\begin{array}{l}\text { Achievable within } \\
\text { current boundaries }\end{array}$ & $\begin{array}{l}\text { (Haughey, 2014, } \\
\text { Gregson, } \\
\text { 2017) }\end{array}$ \\
\hline Time-bound & End dates defined & (Haughey, 2014) \\
\hline Review & Review dates defined & (Haughey, 2014) \\
\hline Objectives & $\begin{array}{l}\text { What and not how } \\
\text { to achieve }\end{array}$ & $\begin{array}{l}\text { (Johnson, 2003, } \\
\text { Gregson, } \\
\text { 2017) }\end{array}$ \\
\hline $\begin{array}{l}\text { Action- } \\
\text { oriented }\end{array}$ & $\begin{array}{l}\text { Actionable items are } \\
\text { preferred over } \\
\text { studies }\end{array}$ & (Johnson, 2003) \\
\hline Non-blaming & $\begin{array}{l}\text { Focus on problems, } \\
\text { not individuals }\end{array}$ & $\begin{array}{r}\text { (Johnson, 2003, } \\
\text { Dekker, 2016) }\end{array}$ \\
\hline
\end{tabular}

*Indicative literature references. 
Table 2. Scope of safety recommendations*.

\begin{tabular}{|c|c|c|}
\hline Dimension & Category & Brief explanation \\
\hline \multirow[t]{3}{*}{$\begin{array}{l}\text { Aspect of } \\
\text { operations }\end{array}$} & $\begin{array}{l}\text { Process } \\
\text { structure }\end{array}$ & $\begin{array}{l}\text { Oriented to low-level } \\
\text { tasks (Re)design of } \\
\text { system's architecture } \\
\text { and functionality }\end{array}$ \\
\hline & $\begin{array}{l}\text { Culture } \\
\text { context }\end{array}$ & $\begin{array}{l}\text { Change of norms and } \\
\text { behaviours }\end{array}$ \\
\hline & & $\begin{array}{l}\text { Focus on politics and } \\
\text { the society }\end{array}$ \\
\hline \multirow[t]{3}{*}{$\begin{array}{l}\text { Stakeholders } \\
\text { affected }\end{array}$} & Macro-level & $\begin{array}{l}\text { Governments, } \\
\text { associations etc. }\end{array}$ \\
\hline & Meso-level & Industry sector(s) \\
\hline & Micro-level & $\begin{array}{l}\text { Organizations and } \\
\text { individuals }\end{array}$ \\
\hline \multirow[t]{3}{*}{$\begin{array}{l}\text { Degree of } \\
\text { renewal }\end{array}$} & Repair & $\begin{array}{l}\text { Short fixes of local } \\
\text { problems }\end{array}$ \\
\hline & Adaptation & $\begin{array}{l}\text { Improvement of larger } \\
\text { systems }\end{array}$ \\
\hline & Innovation & $\begin{array}{l}\text { Creation of new } \\
\text { solutions }\end{array}$ \\
\hline
\end{tabular}

*Adapted from ESREDA (2015).

Table 3. Expected effectiveness of safety recommendations.

Type of control

introduced* Brief explanation

\begin{tabular}{ll}
\hline Physical & Prevent completely actions or access \\
Functional & Use of technology to limit actions \\
Symbolic & $\begin{array}{r}\text { Means to alert for hazards or } \\
\text { remind/train rules, procedures etc. }\end{array}$ \\
Incorporeal & Strategies, general policies, legislation \\
\hline
\end{tabular}

*In descending order of robustness (Hollnagel, 1999).

\section{METHODOLOGY}

Following the establishment of the theoretical framework about the design and classification of safety recommendations as presented above, the researcher aimed at assessing the degree to which the aspects of the framework are known and/or applied by safety investigators and professionals involved in the formulation of safety recommendations in general (e.g., safety and risk managers). The aspects of the framework comprised the topics of a questionnaire that was administered to safety managers, investigators and professionals. The data collected from the analysis with the tool and the questionnaire responses were statistically processed to obtain an overall picture and examine differences across various variables.

\subsection{Survey questionnaire}

The survey instrument was designed with the goal to capture the following information from practitioners involved in the generation of safety recommendations: whether they are knowledgeable of the design criteria and the degree to which they apply those in daily practice along with possible reasons; perceptions about the order of effectiveness of the control types referred in literature, frequency of proposing each control type as part of their role, and explanations about the latter; extent to which they focus on each of the categories included in the three dimensions of recommendations' scope and respective reasons.

The questionnaire included an introductory section where the background and aims of the study were stated along with the voluntary character and anonymity of participation. Also, the particular section referred to the estimated time investment (i.e. up to 15 minutes) and the contact details in the case that the respondents wanted to provide feedback on the questionnaire, get informed about the results of the study or raise any other inquiry.

To examine possible variations of the responses against characteristics of the sample, the subjects were asked to fill in their main job role at the time of participation (i.e. safety manager/officer, safety investigator, or other), the year they started getting involved actively in the generation of safety recommendations, the country they were practicing their vocation at the time of participation, and their highest level of education (i.e. High School, Associate Degree, Bachelor degree, Master degree, Doctoral level, and other).

For each of the design criteria for safety recommendations, the respondents were asked to state whether they know the criterion (possible choices: YES or NO), the extent to which they apply the criterion when creating safety recommendations (possible choices: $0-20 \%, 21-40 \%, 41-60 \%, 61-80 \%$, and $81-100 \%$ ), and explanations about their latter. Regarding the expected effectiveness of recommendations, the corresponding section provided a brief description and a few examples for each of the control types (i.e. physical, functional, symbolic and incorporeal) and asked the participants to rank the controls in the order of their effectiveness, state which control type they most frequently introduce in their safety recommendations, and justify their last answer. It is noted, that the control types were presented to the participants in a random order of effectiveness outlined in the literature (Hollnagel, 1999). The last section of the instrument referred to the scope of recommendations and included a short description for each of the dimensions (i.e. aspects of operations, stakeholders affected and degree of renewal) and their values (see Table 2). The subjects were prompted to choose the frequency to which their recommendations focus on each of the categories of the three dimensions (possible choices: $0-20 \%, 21-40 \%, 41-60 \%, 61-80 \%$, and $81-100 \%$ ) and state respective reasons. 
It is clarified that there were no obligatory questions to be answered. The respondents could omit any demographic or safety recommendation related question. The draft version of the survey instrument was sent to four persons with relevant academic and professional background for their review. Following the revision of the questionnaire according to the remarks collected, its final version was designed online with the use of the Qualtrics platform. The functionality of the online questionnaire was tested with the participation of the same four reviewers. The survey instrument was administered through two main channels: (1) personal emails to contact persons of the network of the Aviation Academy of the Amsterdam University of Applied Sciences that covers various aviation organizations worldwide; (2) online messages to practitioners found on the LinkedIn platform and holding relevant positions (e.g., safety managers, safety investigators). Due to time constraints, three working days were devoted to the administration of the questionnaire and a three weeks period was set for the collection of responses.

\subsection{Sample and analysis of questionnaire responses}

In total, 42 questionnaires were filled. Because of the snowball sampling strategy, the author could not have any information about the number of persons whom the instrument finally reached (e.g., unmonitored or unread emails and messages). Therefore, the response rate could not be estimated. Nevertheless, since the scope of the whole study was an initial assessment of the situation around safety recommendations, the number of responses was deemed as sufficient. Table 4 presents the distribution of the sample across its demographic characteristics. It is clarified that the apart from the main job role, the

Table 4. Distribution of the sample.

\begin{tabular}{llll}
\hline $\begin{array}{l}\text { Demographic } \\
\text { variable }\end{array}$ & Values & $\begin{array}{l}\text { Sample } \\
\text { size }\end{array}$ & $\begin{array}{l}\text { Valid } \\
\text { percentage* }\end{array}$ \\
\hline Main job role & $\begin{array}{l}\text { Safety staff } \\
\text { Safety } \\
\text { investigator }\end{array}$ & 18 & 42.9 \\
& Other & 11 & 31.0 \\
& $<=4$ & 10 & 26.1 \\
Years involved & $<-0$ \\
in generation & 5-11 & 11 & 27.5 \\
of recommend- & $12-18$ & 10 & 25.0 \\
ations & $>=19$ & 9 & 22.5 \\
Geographical & Europe & 30 & 71.4 \\
region & Other & 12 & 28.6 \\
Highest level & $<=$ Bachelor & 18 & 43.9 \\
of education & $>=$ Master & 23 & 56.1 \\
received & & & \\
\hline
\end{tabular}

*Some demographic questions were not answered. rest of the demographics were grouped due to the small number of responses in some of the categories.

Regarding the analysis of data, the frequencies for each the closed questions were calculated to offer an overall view of the responses. Fisher's exact tests were performed to reveal any associations of the knowledge of design criteria with the variables of Table 4. The same variables were also used to conduct Kruskal-Wallis or Mann-Whitney tests (i.e. depending on the number of categories of each variable) for the closed questions which corresponded to ordinal data (i.e. frequency of application of design criteria, frequency of focus of recommendations on the areas defined, and level of effectiveness of controls the recommendations introduce).

It is noted that, to allow the execution of statistical tests, the frequency choices were translated to ordinal figures as follows: $1: 0-20 \%, 2: 21-40 \%, 3: 41-60 \%, 4$ : $61-80 \%$, and $5: 81-100 \%$. The tests were run with the SPSS software version 22 (IBM, 2013). The function of Monte Carlo Exact Test under the settings Confidence Level: 99\% and Number of Samples: 10.0000 was chosen to strengthen the validity of the results. The level of statistical significance was set to 0.05 .

The open-ended questions concerned, a thematic analysis was performed. The researcher individually performed a coding of the answers, which was afterwards tested for reliability with two other colleagues who were not involved in the study. The comments of the raters indicated areas of disagreement as well as cases that the content of the answers had not been captured by the initial codes. Based on these remarks, the coding was revised and retested, resulting in agreement levels ranging from $77 \%$ to $92 \%$ between the researcher and each of the participants as calculated with Cronbach Alpha tests. The finalization of the list of coding themes was followed by the calculation of frequencies per code for each of the questionnaire topics.

It is clarified that in several qualitative responses the subjects did not provide explanations about their choices in the closed questions, but they restated the latter or made general comments not applicable to the particular question. These cases were excluded from the analysis. Due to the small number of valid responses, no statistical tests were conducted between the responses and the demographic characteristics of the participants.

\section{RESULTS}

\subsection{Results from analysis of closed questions}

The frequencies that the design criteria of safety recommendations are known and the extent to which are applied by the survey participants are shown in Table 5. The Non-blaming, Assigned and Realistic criteria were the ones most known, whereas the Review date and Actions criteria were 
Table 5. Design criteria for safety recommendations.

\begin{tabular}{lll}
\hline Criterion & $\begin{array}{l}\text { Median rank } \\
\text { (application) }\end{array}$ & $\begin{array}{l}\text { Frequency (\%) the } \\
\text { criterion is known }\end{array}$ \\
\hline Specific & 4.5 & 90.5 \\
Measurable & 4.0 & 87.8 \\
Assigned & 5.0 & 97.6 \\
Realistic & 5.0 & 95.2 \\
Time-bound & 4.0 & 90.5 \\
Review & 4.0 & 73.8 \\
Objectives & 4.0 & 85.4 \\
Actions-oriented & 4.0 & 75.6 \\
Non-blaming & 5.0 & 100.0 \\
\hline
\end{tabular}

the ones that were least known by the respondents. Also, Spearman's bivariate correlations were performed between the figures of knowledge percentage and the medians. The results of the particular statistical test showed a significant and strong association $(\mathrm{N}=9, \mathrm{rs}=0.870, \mathrm{p}=0.002)$, meaning that the higher the knowledge on a specific criterion, the higher the degree of its implementation.

The Fisher Exact tests between the design criteria and the demographics of the population resulted in significant results only for the association of years of experience in the generation of safety recommendations with the knowledge of the criteria Realistic $(\mathrm{N}=40, \mathrm{p}=0.046)$ and Timebound $(\mathrm{N}=40, \mathrm{p}=0.008)$. The participants with 19 or more years of experience in safety recommendations declared less frequently that they knew about both criteria compared to participants having fewer years of experience. Regarding the frequency of application, the Objectives criterion was applied less frequently by safety managers/officers than the rest of the job roles $(\mathrm{p}=0.039)$ and it was utilized more by the participants with increased years of involvement in safety recommendations generation $(\mathrm{p}=0.025)$.

Regarding the scope of recommendations, the respective medians are reported in Table 6 . The statistics revealed that culture-focused recommendations were applied more frequently by participants with roles other than safety staff and investigators $(p=0.046)$. Recommendations addressed to industry sectors (i.e. meso level) were generated by safety investigators more than other job holders $(\mathrm{p}=0.002)$. Meso - and macro-level types of recommendations were made more frequently by subjects working in Europe $(p=0.044$ and $p=0.021$ respectively) or having a high educational background ( $\mathrm{p}=0.008$ and $\mathrm{p}=0.004$ correspondingly).

The expected effectiveness of safety recommendations concerned, Table 7 presents the survey findings with regard to the perceived degree of effectiveness for each type of control introduced through recommendations. The statistics showed
Table 6. Distribution of scope areas of generated recommendations.

\begin{tabular}{cll}
\hline Dimension & Category & $\begin{array}{l}\text { Median rank } \\
\text { (application) }\end{array}$ \\
\hline Aspect of & Process & 4.0 \\
operations & Structure & 3.0 \\
& Culture & 2.0 \\
Stakeholders & Context & 2.0 \\
affected & Macro level & 2.0 \\
& Meso level & 3.0 \\
Degree of & Micro level & 4.5 \\
renewal & Repair & 4.0 \\
& Adaptation & 4.0 \\
& Innovation & 2.0 \\
\hline
\end{tabular}

Table 7. Perceived effectiveness of safety recommendations.

\begin{tabular}{ll}
\hline Type of control introduced & Median rank \\
\hline Physical & 2.0 \\
Functional & 2.0 \\
Symbolic & 3.0 \\
Incorporeal & 3.0 \\
\hline
\end{tabular}

no associations of the perceived effectiveness with the demographic characteristics of the sample.

\subsection{Results from analysis of open-ended questions}

The codes derived from the thematic analysis and their frequencies showed that in many cases the subjects applied the design criteria because they were mentioned in internal or external documentation or had been seen as best practice. It was widely recognized that the Specific criterion minimizes ambiguity in the implementation of safety recommendations, which according to a few respondents is expected at some degree. However, 3 out of the 20 participants declared that some flexibility is required and recommendations should not be always too specific.

The Measurable criterion was seen by most of the subjects as often unfeasible and a few respondents stated that the effect of changes is more important than their measurement, monitoring does not apply to simple recommendations, and a customization of relevant metrics to each organizational level/function is necessary. About the Assigned criterion, 3 out of the 18 participants argued that recommendations might require the engagement of more than one responsible persons, departments, agencies etc. The comments made about the Realistic criterion showed that this helps in increasing the credibility and feasibility of the recommendation and demonstrating an achieve- 
ment of a balance between the resources required for its realization and the anticipated benefits. However, 5 out of the 23 answers pointed that the Realistic criterion might be difficult to meet due to the diversity of perspectives and interests of the stakeholders involved.

The Time-bound criterion concerned, the participants expressed a variety of views. Four persons recognized that the specific criterion would ensure the implementation of a recommendation, whereas three persons stated their reservations about the feasibility of the criterion. Also, two persons did not know the particular criterion and one person did not contemplate it as important. A similar picture was observed in the answers regarding the knowledge and feasibility of the Review date and Objectives criteria. The latter criterion was appreciated by three respondents because it allows flexibility in the operationalization of the recommendation.

A combination of actions and studies depending on the type of the problem to be solved was proposed by most of the participants. Four out of the 17 subjects argued that the Action criterion increases the feasibility of a recommendation. The application of the Non-blaming criterion was seen as positive by most of the participants regarding the effects on the overall culture and increase of the effectiveness of safety recommendations. Only 1 out of the 23 answers suggested that a focus on individuals/teams might be appropriate in cases of repeated unsafe behaviors.

The answers regarding the type of control most frequently introduced by the survey respondents showed a preference in symbolic controls, followed by incorporeal, functional and physical ones. The explanations given emphasize on the decreased feasibility of controls of technical nature (i.e. physical and functional) due to the demands for more resources for their realization. The views about the effectiveness of technical or non-technical controls (i.e. symbolic and incorporeal) were almost evenly distributed. In three cases, the respondents argued that symbolic and incorporeal controls are more vulnerable and require more frequent repairs. One respondent claimed that the focus on symbolic controls sources from the pressure of authorities who ask for more and better procedures.

Regarding the focus of recommendations on specific aspects of operations, 4 out of the 17 answers addressed that the decision depends on the context of the problem identified and four of the respondents answered that is a matter of organizational focus. The views about the process and culture aspects of operations were divided into two equal parts. Four subjects claimed that those aspects are difficult to change whereas other four subjects stated that the specific aspects are easier and faster to change. Concerning the degree of renewal, repairs and adaptations were seen as equally sufficient to deal with problems, partly because of the lower associated costs. Innovations were contemplated by 4 out of the 14 participants as either expensive or not appropriate to be introduced through recommendations, and two participants suggested that the required degree of renewal depends on the problem under examination.

Lastly, regarding the level of affected stakeholders, 8 out of the 17 subjects stated that their focus on the micro level is dictated by the organizational priority to deal with local problems and the difficulty to affect the meso and macro levels. One respondent recognized that micro level interventions are cheap and another respondent stated that interventions at the lowest level could lead to changes to the rest of the levels. Once more, many subjects declared that the type of each problem is as a parameter to decide which stakeholders will be addressed in a recommendation.

\section{DISCUSSION}

The overall results regarding the design of safety recommendations suggest that most of the participants are knowledgeable about the criteria identified in the academic and professional literature and apply these criteria to at least $60 \%$ of the recommendations but with different extents. Rather expectedly, the knowledge of a specific criterion was positively associated with the degree of its application when generating safety recommendations. However, such knowledge was obtained more through experience, best practice or organizational documentation rather than international and regional standards. The lack of guidelines in industry standards might explain partially the fact that some of the criteria were not known and consistently applied by a fraction of the survey participants. The statistical tests showed a few differences across some criteria, mainly linked to the years of experience in generating recommendations. Two of the criteria were less known and another criterion was more frequently applied by participants with higher experience.

The Non-blaming criterion was known and applied by all participants, thus indicating that the necessity for a just culture in aviation (Humpreys, 2014, Michaelides-Mateou and Mateou, 2016, Quinn, 2007) and the role that recommendations can play in realizing a culture of fairness have been well communicated across the particular industry sector. The criteria of Assigned and Realistic also scored very high regarding the level of familiarity of the respondents and the degree of their application, this partially attributed by the participants to the contribution of these criteria to increased credibility of recommendations. On the other hand, the Review and Actions criteria were not 
known by about one-quarter of the survey participants. These criteria were seen as important but sometimes not feasible or binary regarding their application. In general, the respondents expressed concerns about the strict satisfaction of the whole set of criteria for every single recommendation and argued that each case must be handled differently.

Concerning the type of controls introduced through safety recommendations, interestingly, their effectiveness as perceived by the participants is not aligned with the literature suggestions, the results showing a reverse order. Whereas the work of Hollnagel (1999) implies that physical and functional controls are more robust and effective, the respondents viewed the symbolic and incorporeal controls as such. The comments collected showed that the viewpoints about the effectiveness of technology and non-technology based controls were evenly divided. According to the participants, the main factors driving the recommendation of a specific control type are the resources available and the expectations of the authorities for improved procedures. Hence, the author contemplates that the responses about the effectiveness of controls were affected by current practice and not a consideration of their technical characteristics or potential to deal with a hazard or risk more successfully.

Nevertheless, the positions of the participants regarding the types of controls preferred are in tandem with literature suggesting that the amendment of rules or introduction of new ones are favored due to the low costs involved and the timeliness and easiness of implementation (Bourrier and Bieder, 2013). Moreover, the viewpoints of the respondents seem to confirm their efforts to generate recommendations that are realistic by taking into account the existing boundaries (e.g., resources, operational needs).

The findings regarding the scope of recommendations showed that the higher and wider the level of operations, stakeholder and renewal, the less frequency corresponding changes are suggested. Recommendations that focus on the physical processes and the work floor, specific organizations and repair of problems had been more frequently introduced by the participants. On the other hand, interventions at the culture and context aspects of the operational area, suggestions to the regulatory and standardization levels and recommendations for innovative solutions were least frequently generated. The comments of the respondents seemed to agree about the effects of cost and time limitations along with political factors that can discourage the formulation of recommendations addressing wider and deeper systemic flaws. A few conflicting statements were observed regarding the easiness to make process or cultural changes, this indicating somehow misaligned perceptions.
The statistics regarding differences of the recommendations' scope across the demographics included in the study showed that meso-levels of stakeholders (i.e., whole industry sectors) were addressed more frequently by safety investigators. This finding can be explained by the fact that aviation safety investigation standards (e.g., ICAO, 2003, 2011) prompt the examination of latent factors which in turn allows the formulation of recommendations targeted beyond organizational boundaries. Furthermore, recommendations for stakeholders other than specific organizations and individuals were generated more frequently by respondents working mainly in Europe and having a higher level of education. These findings might reflect the effects of different regional and national cultures (e.g., ICAO, 2013, Stolzer et al. 2008) as well as an influence of educational breadth and depth on the tendency to adopt systemic views and address deficiencies at higher system levels.

\section{CONCLUSIONS}

The current study was a first initiative to map the situation in the aviation industry around the engineering of safety recommendations. Regarding the degree to which professionals are knowledgeable about the design criteria that can increase the quality and effectiveness of recommendations and the extent to which apply these criteria in practice, the results suggested an adequate level of knowledge and a satisfactory frequency of application of the nine design criteria included in the research. However, the findings were attributed more to the employment of best practice and the reference of such criteria in organizational documentation, rather than to guidelines from regional or international guidelines or topics of training. Hence, the inclusion of respective material in standards and safety management/investigation courses is highly recommended to achieve consistency in the generation of safety recommendations and establish a commonly referred framework for their design. Nonetheless, such a framework must be viewed as a guide and not a compliance-check reference. As the study participants stated, each case has a different context and the satisfaction of all design criteria might not be feasible or proper for all recommendations.

The findings concerning the classification of recommendations based on the types of controls they introduce showed a gap between perceptions of the practitioners and the suggestions of the literature. The former viewed "soft" control types as more effective than "hard" ones, whereas literature implies the opposite. It is noted that the categorization of controls does not aim at stating a preference of any type over another but rendering the industry 
aware of the weaknesses and strengths of each control type. Nonetheless, the conduction of further studies is suggested as a means to provide empirical evidence about the actual level of effectiveness of each type of control and indicate any optimum combinations of those.

When considering the focus areas of recommendations, the findings suggested an emphasis on the repair of lowest activity levels rather than systemic interventions and innovative solutions. A common characteristic of all topics examined in this study was the influence of resource and political boundaries on the generation of safety recommendations, which usually lead practitioners in suggesting cheap and easy to implement fixes. From a pragmatic viewpoint, the situation mentioned above is somewhat unavoidable, but this should not exclude the visible and explicit justification of choices when engineering safety recommendations. The recognition and documentation of the boundaries imposed on each case can support the aggregation of data and their monitoring to inform stakeholders accordingly and possibly lift existing limitations when situations allow.

The researcher would like to point out that the small sample of the current study does not allow to claim generalization of the results. However, the finding of this research can trigger the execution of similar studies at larger scales at regional or international levels regardless of industry sector.

\section{REFERENCES}

Bourrier, M. \& Bieder, C. 2013. Trapping Safety Into Rules: How Desirable or Avoidable is Proceduralization? Farnham: Ashgate.

De Vos, B. 2016. Evaluation the Quality of Recommendations Formulated in Accident Reports. BSc graduation thesis (unpublished). Amsterdam: Aviation Academy, Amsterdam University of Applied Sciences.

Dekker, S. 2016. Just culture: restoring trust and accountability in your organization. (3rd ed.). Boca Raton: CRC Press.

ESReDA. 2015. Case study analysis on dynamic learning from accidents. Retrieved from European Safety, Reliability \& Data Association: http://www.esreda.org

Gregson, M. 2017. What Makes a Good Air Safety Recommendation? ISASI Forum, October-December 2017, pp. 15-17
Haughey, D. 2014. A Brief History of SMART Goals Retrieved from Project Smart: https://www.projectsmart.co.uk/brief-history-of-smart-goals.php.

Hollnagel, E. 1999. Accidents and Barriers. Retrieved from University of Linköping, Sweden: http:// www.hhs.iup.edu/CJANICAK/SAFE541CJ/ Barrier $\% 20$ Analysis $\% 20$ Paper.pdf.

Humpreys, K. 2014. Just Culture: Can there be a Just Culture in Aviation Safety Occurrence Reporting Systems. Technical paper, Adelaide: ISASI 2014 Seminar.

IBM. 2013. SPSS Statistics for Windows. Version 22.0. New York: IBM Corp.

ICAO. 2003. Training Guidelines for Aircraft Accident Investigators. Cir 298 AN/172. Canada: International Civil Aviation Organization.

ICAO. 2008. Hazards at aircraft accident sites. Circular 315. Montreal: International Civil Aviation Organization.

ICAO. 2011. Manual on Accident and Incident Investigation Policies and Procedures. Doc 9962. Montreal: International Civil Aviation Organization.

ICAO. 2013. Safety Management Manual. Doc. 9859. Montreal, Canada: International Civil Aviation Organization.

ICAO. 2015. Manual of Aircraft Accident and Incident Investigation. Doc 9756 AN/965. Part I Organization and Planning, 2nd. Montréal: International Civil Aviation Organization.

Johnson, C. 2003. Failure in Safety-Critical Systems: A Handbook of Accident and Incident Reporting. Glasgow: University of Glasgow Press.

Kiefer, K. 2016. Quality Evaluation of Safety Recommendations Formulated in Aircraft Accident Reports, BSc graduation thesis (unpublished). Amsterdam: Aviation Academy, Amsterdam University of Applied Sciences.

Michaelides-Mateou, S. \& Mateou, A. 2016. Flying in the Face of Criminalization: The Safety Implications of Prosecuting Aviation Professionals for Accidents. Oxon: Routledge.

Pooley, E. 2013. Harmonisation of safety recommendations. Technical paper. Madrid: ESASI 2013.

Quinn, K.P. 2007, December. Battling Accident Criminilization. Aerosafety World: 11-12.

Stolzer, A.J., Halford, C.D. \& Goglia, J. 2008. Safety Management Systems in Aviation. Aldershot: Ashgate.

Zonneveld, J. 2016. Assessment of Safety Recommendations, BSc graduation thesis (unpublished). Amsterdam: Aviation Academy, Amsterdam University of Applied Sciences. 